\title{
Metamaterial-based high efficiency portable sensor application for determining branded and unbranded fuel oil
}

\author{
MEHMET ALI TÜMKAYA ${ }^{1}$, MUHARREM KARAASLAN ${ }^{1}$ and CUMALI SABAH ${ }^{2,3, *}$ \\ ${ }^{1}$ Department of Electrical and Electronics Engineering, İskenderun Technical University, Iskenderun, Turkey \\ ${ }^{2}$ Department of Electrical and Electronics Engineering, Middle East Technical University-Northern Cyprus Campus \\ (METU-NCC), Kalkanli, Guzelyurt, TRNC/Mersin 10, Turkey \\ ${ }^{3}$ Kalkanli Technology Valley (KALTEV), Middle East Technical University-Northern Cyprus Campus (METU-NCC), \\ Kalkanli, Guzelyurt, TRNC/Mersin 10, Turkey \\ *Author for correspondence (sabah@metu.edu.tr)
}

MS received 17 March 2017; accepted 15 December 2017; published online 4 July 2018

\begin{abstract}
In this study, we have developed an efficient metamaterial (MTM)-based portable fuel oil sensor in order to distinguish branded and unbranded fuel-oil samples. Electromagnetic properties of the fuel-oil samples are experimentally obtained and these data are defined in numerical analysis to design and test the performance of MTM sensor. Then, simulated MTM-based sensor structure is fabricated and measured to observe the efficiency and agreement to numerical results. Numerical and experimental studies are conducted in the microwave frequencies of 10-11 GHz. The experiment results are in accordance with the simulation ones. It can be seen from the results that the proposed MTM-based sensor can be implemented into the sensor industry for distinguishing the branded and unbranded fuel oil for several frequency regions.
\end{abstract}

Keywords. Metamaterials; sensors; fuel oil sensing; portable branded and unbranded gasoline sensors.

\section{Introduction}

There is a continuous interest on metamaterials (MTMs), which have unique and unusual electromagnetic (EM) properties not found in nature. These behaviours of MTMs have lead the science community to conduct researches in many application areas, including sensors [1], absorbers [2], super lenses [3], frequency shifter [4,5], polarization rotator $[2,6]$ etc. in various industries. MTMs consist of periodically arranged subwavelength resonators and are used in almost every part of the EM spectrum from seismic to optic regions [7].

MTM-based sensors are getting more and more attention due to their stable behaviours, more efficient sensing abilities and low manufacturing, adjusting, measurement and implementation costs. With the developing technology, we will most probably see more and more MTM-based devices in our daily life. Application-dependent subgroups of bio/chemical sensors [8-13], strain sensors [14-19] and thin-film sensors [20-22] can be recognized within this main group.

The motivation of this study is to investigate and determine the difference between branded and unbranded gasoline samples, which is achieved by creating a new MTM structure. Electromagnetic properties of registered and unregistered fuel oil samples are compared and evaluated. Dielectric and loss tangent parameters of the samples are obtained. These parameters of the samples are determined in a lab environment. Obtained results for dielectric variation and the loss tangent are used in the numerical analyses for X-band frequency regime. In order to achieve an efficient sensing behaviour, the frequency range should be chosen so that dielectric constant has a linear curve in that particular frequency range. $\mathrm{X}$-band meets that requirement perfectly and the applications from communication to military radars in X-band can also make this band more appropriate for proposed sensor. In order to show the working efficiency of the proposed sensor application, it is tested by using scattering parameters. This efficiency depends on the sample filled into the sensing layer; reflected waves in both waveguide ports show various resonances allowing us to establish the sensing characteristic. The experiment results are in good agreement with the simulation ones. It shows that the proposed model can be used for determination and monitoring the usage of branded and unbranded gasoline as a portable.

\section{Numerical design and fabrication of MTM-based high efficiency portable sensor}

Proposed MTM-based sensor unit cell has a simple structure design. It consists of a rectangular frame covering a rectangular horizontal microstrip line placed on a dielectric substrate, which is chosen as FR4. FR4 is chosen due to its low cost and its relatively low loss EM properties. It is generally used as an electrical insulator possessing remarkable 


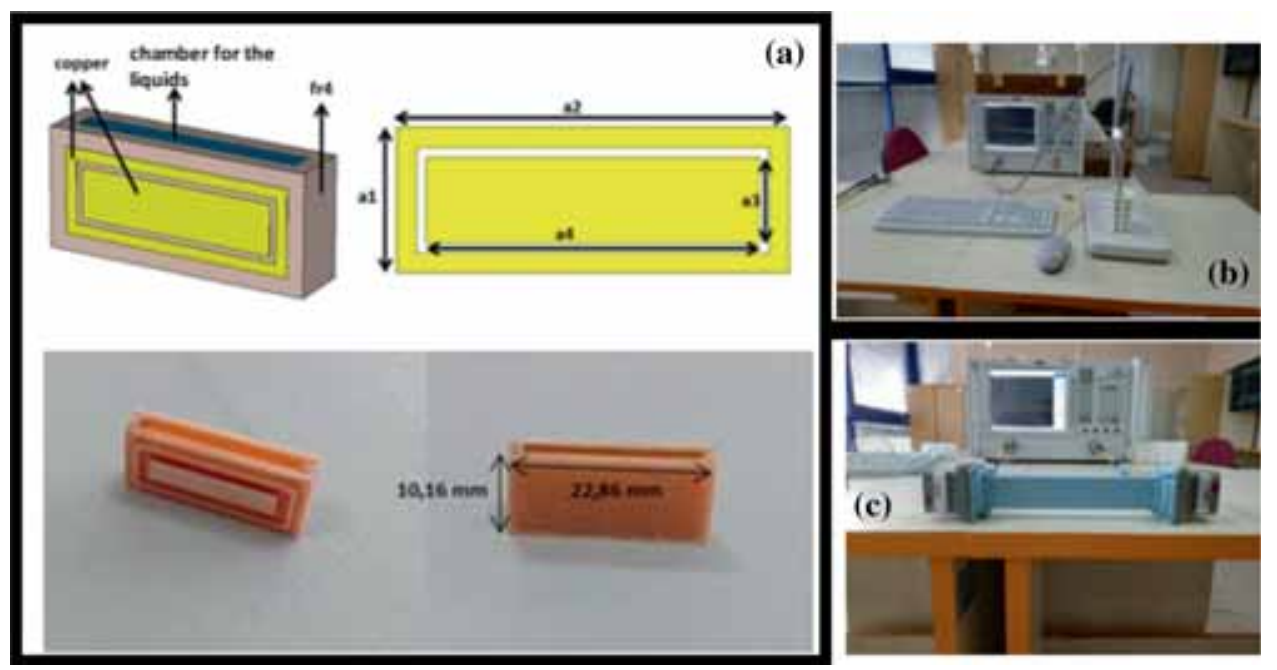

Figure 1. (a) Fabricated sensor design, (b) dielectric measurements setup and (c) waveguide measurements setup.

mechanical strength for printed circuit boards. It has thickness of $1.6 \mathrm{~mm}$, relative permittivity of 4.2 , magnetic permeability of 1 and loss tangent of 0.02 values. Suggested sensor structure includes a sensing layer whose three edges are closed with metallic boundary/PEC and the top edge is left open to provide wave propagation. The sensing layer is filled with the fuel-oil sample in order to carry out the EM tests to observe sensing phenomena. For the conducting part of the structure, we have chosen copper material, which has an electrical conductivity of $5.8001 \times 10^{7} \mathrm{~S} \mathrm{~m}^{-1}$ and a thickness of $0.035 \mathrm{~mm}$. The backside of the structure does not include any copper metal plate. The dimension of the proposed design is compatible for X-band waveguide. Dimension of gap between inner and outer resonators is $1 \mathrm{~mm}$. Width and length of the FR4 is $22.86 \mathrm{~mm}$ and $10.16 \mathrm{~mm}$, which are proper to the dimensions of X-band waveguide. Figure 1 shows general view of proposed sensor design, dimensions of the structure, fabricated sample and measurement setup.

The dimensions of the geometry of the proposed sensor are defined according to the numerical and parametric study results. A parametric study is considered to obtain optimal structure dimensions of the proposed sensor design by Finite Integration Technique (FIT) based EM simulation software. The small rectangular line in the frame is studied parametrically and the dimensions for the optimum sensing results are found as a1 $=7.3 \mathrm{~mm}$, a $2=20 \mathrm{~mm}$, a $3=4.3 \mathrm{~mm}$, $\mathrm{a} 4=17 \mathrm{~mm}$, whose locations are shown in figure 1a. Proposed design improves the coupling effect between inner and outer resonators, which is directly related with the sensitivity exactitude level for sensor application. After the numerical studies, we fabricated the suggested MTM-based sensor design by using the printed circuit board technique as shown in figure 1a. The LPKF protomat is used for fabrication of sensor structure.

\section{Experimental setup for fuel-oil samples and MTM-based high efficiency portable sensor}

In this part, numerical and experimental setup for fuel-oil samples and MTM-based high efficiency portable sensor is realized and analysed as detail. Firstly, in order to design a sensor for sensing the fuel-oil types, the EM parameters of the samples are extracted and obtained. Secondly, fabricated MTM-based high efficiency portable sensor is tested to determine reflection and transmission properties of the structure.

Dielectric constants and loss tangent of the samples are gathered by using KEYSIGHT brand PNA-L N5234 Network Analyzer, which works up to $43 \mathrm{GHz}$ frequency range. Figure $1 \mathrm{~b}$ shows dielectric measurements setup.

PNA-L N5234 Network Analyzer includes a dielectric probe and related software allowing to monitor the EM properties of any liquid in the working frequency band. Measurement of dielectric properties involves measurements of the complex relative permittivity $\left(\varepsilon_{\mathrm{r}}\right)$ and complex relative permeability $\left(\mu_{\mathrm{r}}\right)$ of the materials. The open-ended coaxial probe method is used to determine samples' EM properties. A complex dielectric permittivity consists of a real and an imaginary part. The real part of the complex permittivity, also known as dielectric constant, is a measure of the amount of energy from an external electrical field stored in the material. The imaginary part is zero for lossless materials and is also correlated with loss factor. It is a measure of the amount of dissipated energy in the material due to an external electric field component of applied wave. The term is called loss tangent and it represents the ratio of the imaginary part to the real part of the complex permittivity. The loss tangent is also called by terms such as tangent loss, dissipation factor or loss factor. The complex permeability also consists of a real part, which represents the amount of energy from an external magnetic field 
Table 1. Dielectric constant and loss tangent values of branded and unbranded fuel-oil samples.

\begin{tabular}{|c|c|c|c|c|c|c|c|c|}
\hline \multirow[b]{2}{*}{$\begin{array}{l}\text { Frequency } \\
(\mathrm{GHz})\end{array}$} & \multicolumn{4}{|c|}{ Reel dielectric constants $(\varepsilon)$} & \multicolumn{4}{|c|}{ Loss tangent (tang. $\delta$ ) } \\
\hline & $\begin{array}{l}\text { Gasoline } \\
\text { (branded) }\end{array}$ & $\begin{array}{c}\text { Gasoline } \\
\text { (unbranded) }\end{array}$ & $\begin{array}{c}\text { Diesel } \\
\text { (branded) }\end{array}$ & $\begin{array}{c}\text { Diesel } \\
\text { (unbranded) }\end{array}$ & $\begin{array}{l}\text { Gasoline } \\
\text { (branded) }\end{array}$ & $\begin{array}{c}\text { Gasoline } \\
\text { (unbranded) }\end{array}$ & $\begin{array}{c}\text { Diesel } \\
\text { (branded) }\end{array}$ & $\begin{array}{c}\text { Diesel } \\
\text { (unbranded) }\end{array}$ \\
\hline 8 & 2.43 & 2.51 & 2.07 & 2.68 & 0.34 & 0.23 & 0.15 & 0.16 \\
\hline 9 & 2.32 & 2.44 & 2.04 & 2.62 & 0.39 & 0.27 & 0.17 & 0.19 \\
\hline 10 & 2.19 & 2.33 & 1.98 & 2.56 & 0.43 & 0.30 & 0.20 & 0.21 \\
\hline 11 & 2.08 & 2.24 & 1.95 & 2.50 & 0.47 & 0.32 & 0.22 & 0.24 \\
\hline 12 & 1.96 & 2.15 & 1.90 & 2.43 & 0.51 & 0.35 & 0.23 & 0.25 \\
\hline
\end{tabular}

stored in the material, whereas the imaginary part represents the amount of energy dissipated due to the magnetic field. Measurement on the complex permeability is only applicable to magnetic materials. Most materials are non-magnetic and thus, the permeability is close to the permeability of free space.

In order to test dielectric and loss tangent values of the fuel-oil samples, measurement setup is installed as shown in figure 1b. Extracted dielectric and loss tangent values in $\mathrm{X}$-band frequency regime of the fuel-oil samples are listed in table 1. One can see from the table 1 that branded and unbranded samples show different values of dielectric constant and loss tangent in the same frequencies. This is the core of this study and allows us to build the proposed sensor to detect the sample type under test.

Figure 2 shows alteration of dielectric permittivity of gasoline_branded and gasoline_unbranded and diesel_branded and diesel_unbranded, correspondingly. It can be seen from figure 2 that almost linear variations are observed between samples. This is very important point for sensing operation. Dielectric permittivity variations of the samples are obtained by using a dielectric probe, corresponding software and PNA-L N5234 Network Analyzer.

Before performing the EM measurements using the probe of the network analyzer, it has to be calibrated as explained below. For the calibration, air, water, whose magnetic characteristics are known, and a special calibration tool for short circuit are used. First, the frequency range in which the measurement will be carried out is defined in the network analyzer, which is between 8 and $12 \mathrm{GHz}$ in our case. By entering the temperature of the water used for the calibration into the analyzer, the first step is completed. The air is measured while the dielectric probe is idle in the second step. The probe is then immersed in the water and the short circuit apparatus is placed on top of the probe to finish the calibration. In order to validate the performed calibration, water and air are measured again and the values are examined. Re-measured values of water with dielectric constant of about 80 are compared with the known value and the calibration of the probe is verified. The EM measurements of the specimens are performed after a verified calibration.

As seen from figure 2, dielectric permittivity variations are linear for almost all frequency range. We are working on the differences in the magnitude levels as expected. These differences are the basis of the study and were used to design more efficient sensor for the determination of the diesel and gasoline samples of branded and unbranded types. Reel dielectric constants gasoline_branded and gasoline_unbranded is 2.19 and 2.33 in frequency of $10 \mathrm{GHz}$, respectively. This difference is likely valid for diesel gasoline as 1.98 and 2.56 for diesel_branded and diesel_unbranded, separately. The minimum dielectric constant difference between gasoline samples is 0.1 and diesel samples is 0.65 . Hence, the confirmed minimum difference of dielectric constants is sufficient to determine the type of samples with a MTM-based sensor.

\section{Numerical and experimental results}

The simulation program used in this study is a finite integration technique (FIT) based one and provides threedimensional EM solutions for high frequencies. The FITbased simulation code allows the discrete formulations of Maxwell equations in integral form for computers and provides the opportunity to simulate complex EM field problems [23]. FIT transforms Maxwell equations into a linear equation system. This technique processes the interfaces between the different media more precisely and it is flexible in geometric modelling. MTM sensor structure proposed and used in this study was designed and analysed with this simulation. The program allows the calculation of EM quantities, such as electric fields, surface currents, magnetic fields, power flows, current densities, surface and volume power dissipation densities, electric energy densities and magnetic energy densities.

FIT-based EM simulations are performed for proposed sensor application. Dielectric and loss tangent values of samples, as given in table 1, within the range of 8-12 GHz are defined one by one and used for the numerical analysis in order to obtain and evaluate the scattering parameter changes in both reflection and transmission in terms of $\mathrm{dB}$.

As mentioned before, waveguide and adaptor used for the measurements can be seen in figure 1c. The model number of the waveguide is Maury X101A6. The sample is placed in the waveguide and connected to the analyzer ports via KEYSIGHT brand adaptors with the number of X281A. 

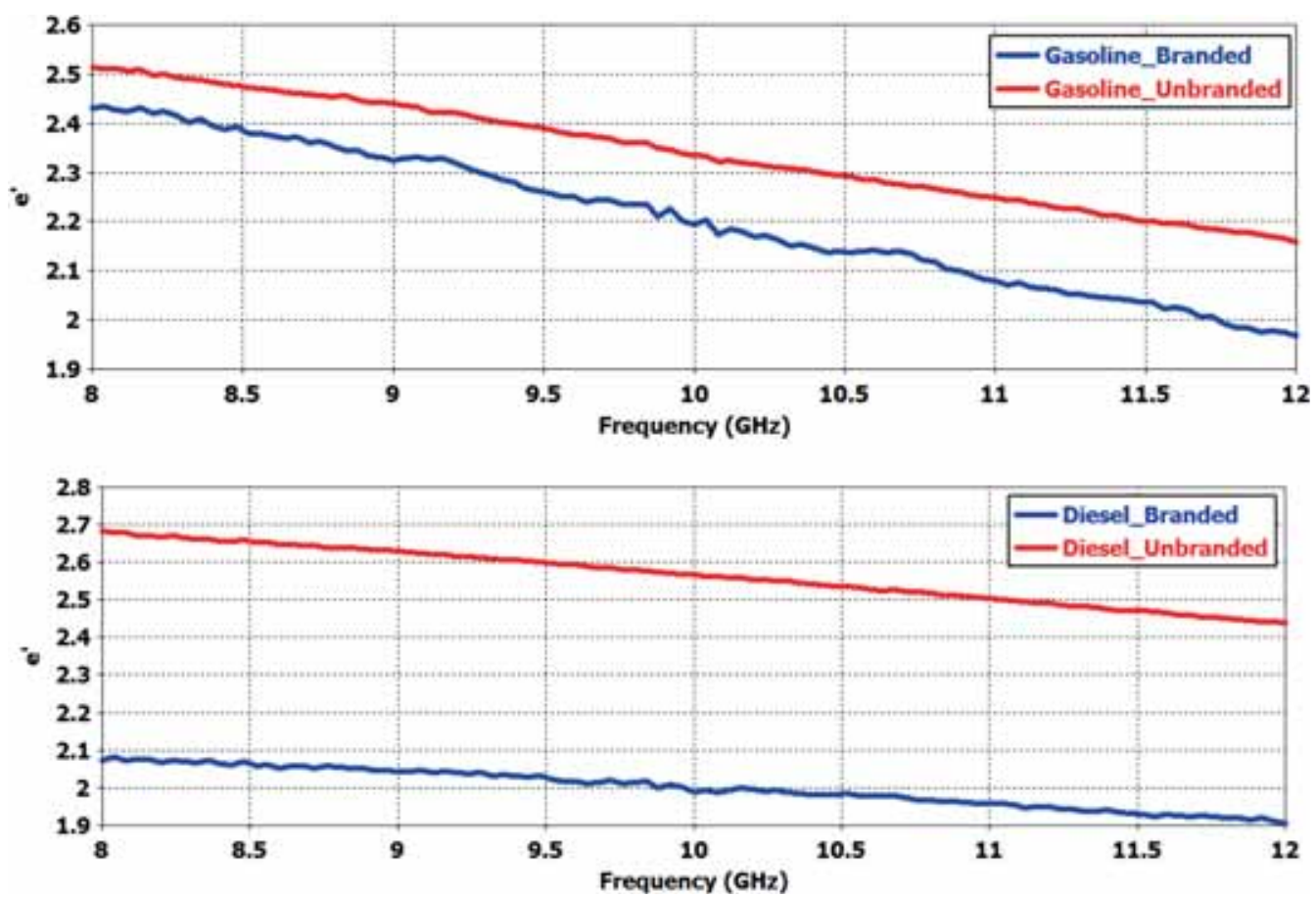

Figure 2. Dielectric values for gasoline and diesel (both branded and unbranded) samples within the range of $8-12 \mathrm{GHz}$.

Scattering parameters are measured when the sensor is in the waveguide with and without gas samples filled into the sensor. In addition, the sensor is also measured when the sensing chamber is empty (filled with air) for calibration and the results are recorded. The measurement setup is shown in figure $1 \mathrm{c}$.

Obtained numerical results are presented and discussed for comparison with the experimental results. Figure 3 shows reflection values of the diesel branded and diesel_unbranded gasoline samples within the range of $10-11 \mathrm{GHz}$ for numerical analysis. It can be seen from figure 3 , resonance frequency values are different for both samples. Resonance frequency shifts left when diesel_unbranded gasoline sample is used. While S22 value of the diesel_unbranded gasoline sample is about $-33 \mathrm{~dB}$ at about $10.268 \mathrm{GHz}$, this value is almost $-29 \mathrm{~dB}$ and shifted right to $10.356 \mathrm{GHz}$ for diesel_branded gasoline sample in numerical results. The resonance frequency shift between two samples is due to the capacitive effect change of the backward reservoir. The higher value of dielectric constant (unbranded diesel) increases capacitance of the reservoir and decreases the resonance frequency of L-C resonant circuit. The reflection coefficient of the unbrandeddiesel is lower due to higher value of loss tangent, shown in table 1. There is an $88 \mathrm{MHz}$ difference between reflection values of samples at the resonance frequency, which is quite enough bandwidth to distinguish them even when they are mixed with each other. In addition, we experimentally tested and verified the characteristic of the proposed sensor by measuring the complex S-parameters as shown in figure 6. For experimental results, while $S 22$ value of the diesel_unbranded gasoline sample is about $-33 \mathrm{~dB}$ at about $10.268 \mathrm{GHz}$, this value is almost $-29 \mathrm{~dB}$ and shifted right to $10.356 \mathrm{GHz}$ for diesel_branded gasoline sample. It is noted that the small differences between the experimental and numerical data are imputed by fabrication tolerances, measurement without sample holder and dielectric dispersion of the FR4-substrate. The misalignment during the experiment may also be considered as a new source of error. The accuracy of the experiment can be well described by the agreement between the numerical and experimental results.

As the following step, the electric field and surface current distributions of diesel_branded and diesel_unbranded gasoline samples are investigated at the resonant frequency of 10.356 and $10.268 \mathrm{GHz}$ for numerical analysis results in order to better understand and show the physical mechanism of the operation principle of the proposed model at the resonances. Obtained results are shown in figure 4. High concentration of the electric field distribution around the sides of structure is observed. The electric field is strongly coupled with the resonators. This coupling provides independent electric response as working like a quadruple for all polarizations. The electric field responses of the resonator for both branded and unbranded diesels are concentrated around all metallic part of the sensing layer. The electric field distribution of branded diesel is higher than unbranded one at the corner parts and penetrates deeper due to the lower real value of dielectric constant. From figure 4, it is observed that there are parallel and anti-parallel current distributions in the pattern 

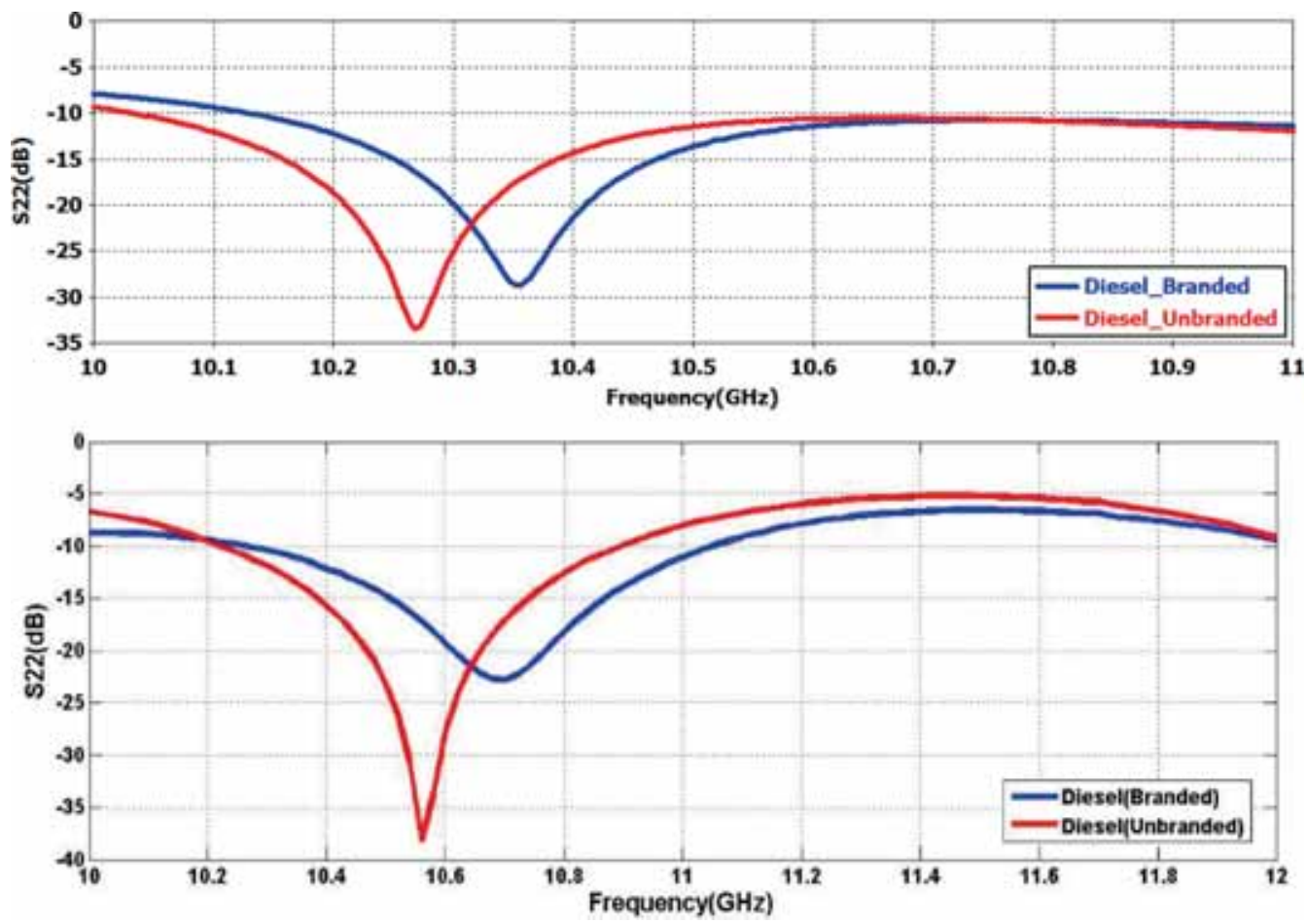

Figure 3. Simulated and measured reflections for diesel_branded and diesel_unbranded gasoline samples within the range of $10-11 \mathrm{GHz}$.
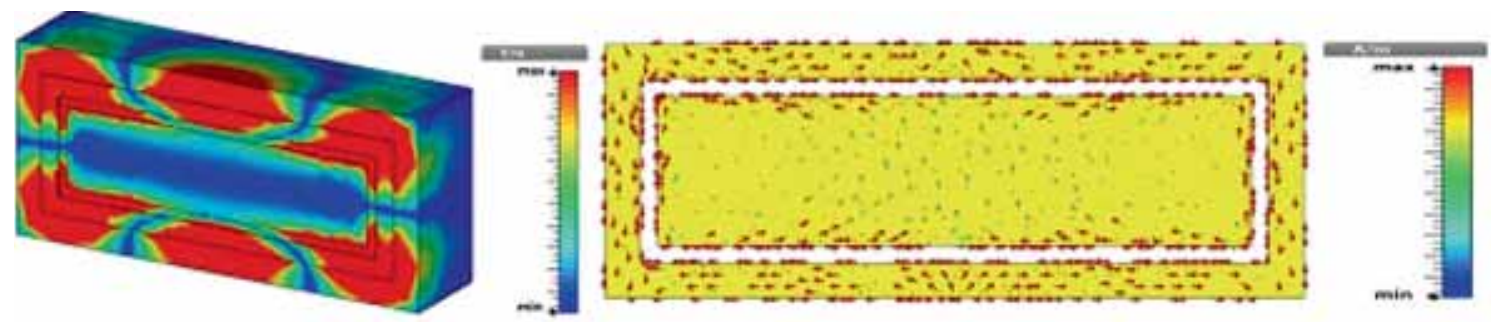

(a)
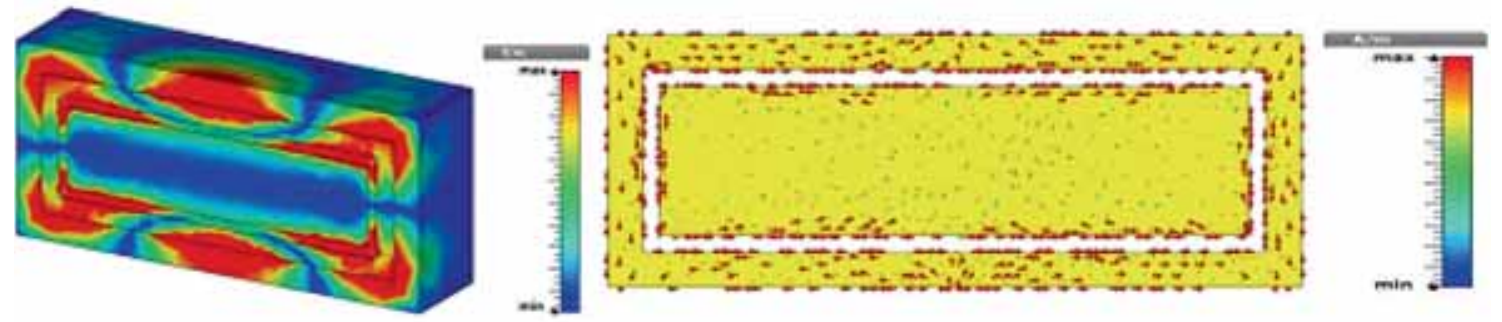

(b)

Figure 4. Simulated electric field and surface current distributions of the proposed sensor filled with (a) diesel_branded and (b) diesel_unbranded gasoline samples.

that is responsible for the magnetic response. The two halves of rectangular metallic resonator behaves as two magnetic resonance circuits. The magnetic loop is complicated on the metallic parts and the space at the centre of the resonator. It is seen that the magnetic resonance of branded diesel is also slightly higher than the unbranded one. However, the magnetic resonances are weak. Consequently, the mentioned currents are driven by strong electric coupling at the resonant frequency.

As a second experiment, the determination of the types of gasoline is investigated both numerically and experimentally in the frequency range of 11 and $12 \mathrm{GHz}$. Numerical 

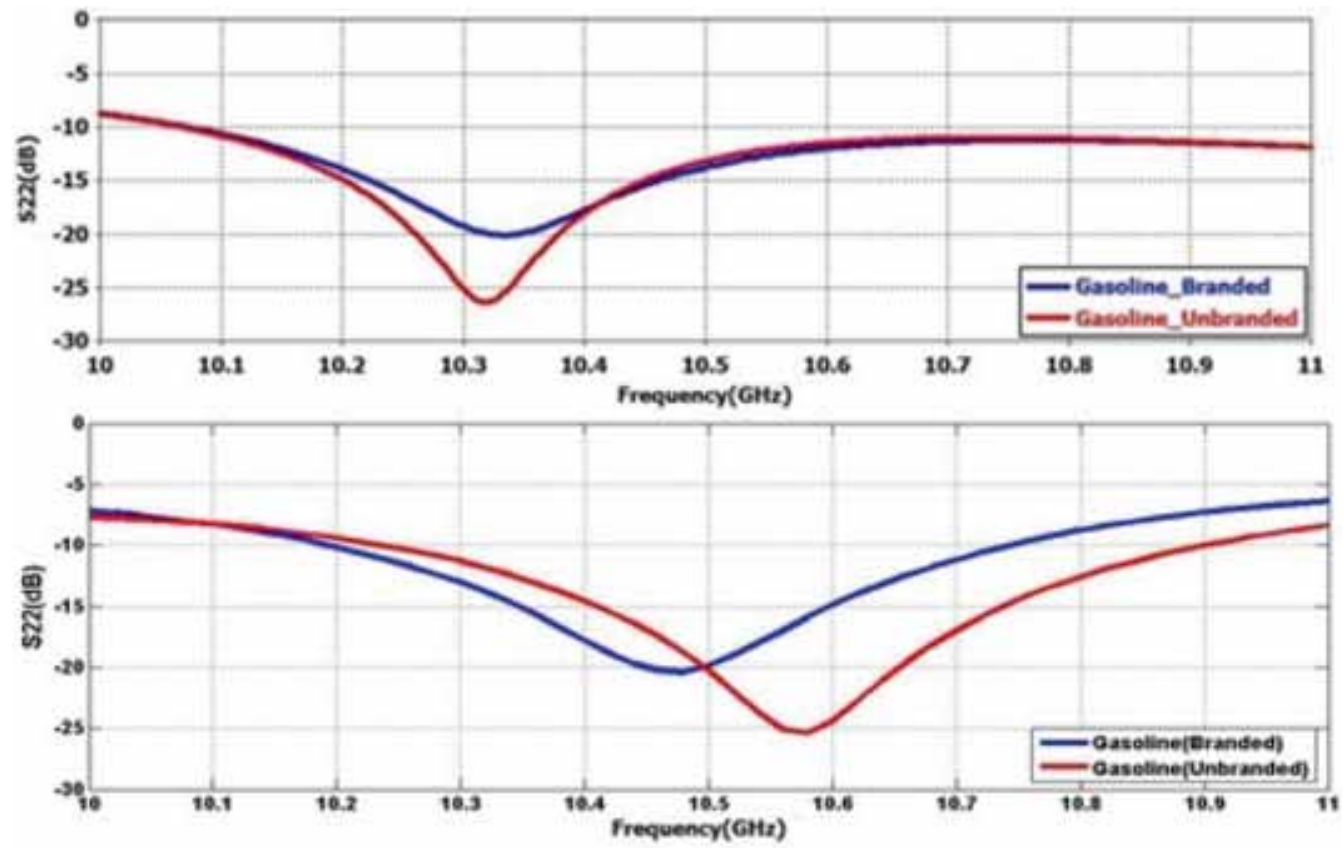

Figure 5. Simulated and measured reflections for the gasoline (both branded and unbranded) samples within the range of $10-11 \mathrm{GHz}$.
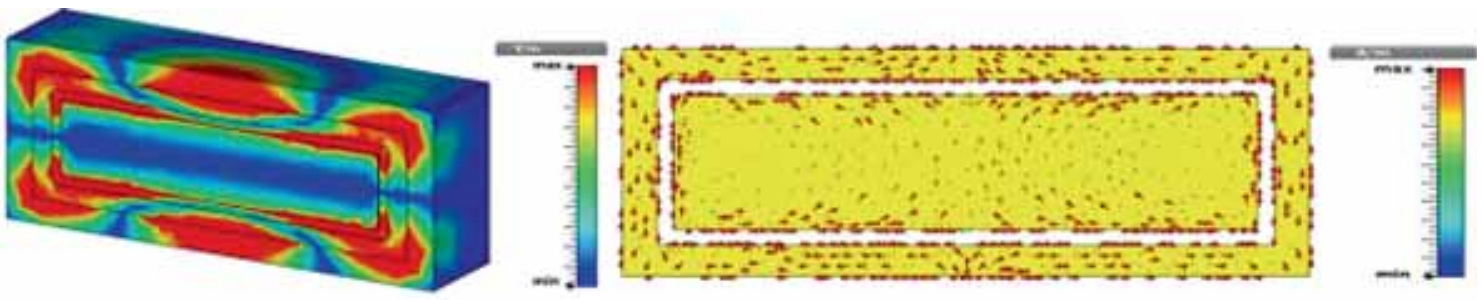

(a)
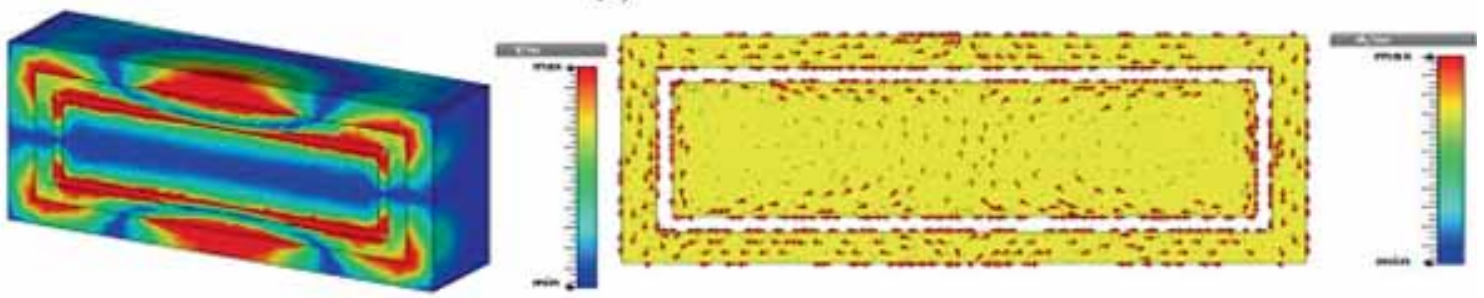

(b)

Figure 6. Simulated electric field and surface current distributions of the proposed sensor filled with (a) gasoline_branded and (b) gasoline_unbranded samples.

and experimental results of the gasoline_branded and gasoline_unbranded gasoline samples are shown in figure 5 . The resonance shift is not much for the gasoline samples, since the real part of dielectric constants of samples are close to each other. The distance of the dielectric constants is only 0.1 . Hence, the resonance frequency shift is smaller with respect to diesel samples. Besides, the magnitude of the reflection parameters (S22) is distinctive due to significant differences of loss tangent. As seen from the numerical results, gasoline_unbranded gasoline sample has a bigger S22
(dB) value than gasoline_branded sample along with a small difference in the resonance frequencies. As can be seen that, the proposed MTM sensor can be used to determine gasoline types with respect to loss factor in a constant frequency.

Resonance frequency shifts to right when gasoline_branded sample is used. While S22 value of the gasoline_unbranded gasoline sample is around $-26 \mathrm{~dB}$ at about $10.32 \mathrm{GHz}$, this value is almost $-20 \mathrm{~dB}$ and shifted right to $10.332 \mathrm{GHz}$ for gasoline_branded gasoline sample in numerical results. There is a $12 \mathrm{MHz}$ difference between reflection values of samples 

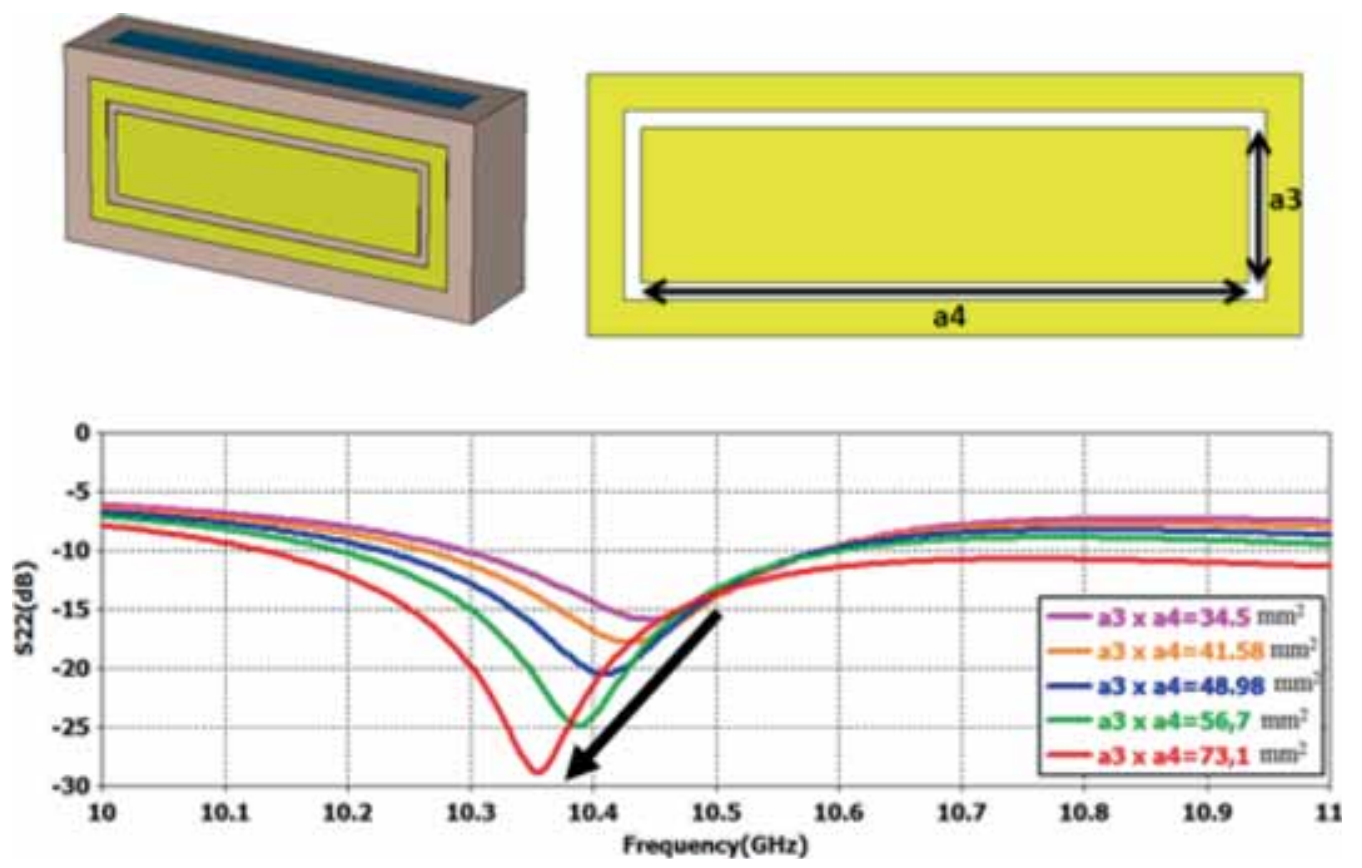

Figure 7. Numerical parametric study S22 (dB) results of MTM-based sensor structure.

at the resonance frequency, which is quite enough bandwidth for us to distinguish them even when they are mixed with each other. In addition, we experimentally tested and verified the characteristic of the proposed sensor by measuring the complex S-parameters as shown in figure 5. For experimental results shown in figure 5, while S22 value of the gasoline_branded gasoline sample is about $-33 \mathrm{~dB}$ at about $10.268 \mathrm{GHz}$, this value is almost $-29 \mathrm{~dB}$ and shifted right to $10.356 \mathrm{GHz}$ for gasoline_unbranded gasoline sample. The inconsistency between measurement and numerical results stems from fabrication errors and lack of sample holder in waveguide for measurement process. Numerical results show that the proposed model can be used successfully to determine the difference between a branded and unbranded fuel oil in terms of their resonance frequencies and their reflection values.

The electric field distributions of the gasoline branded and unbranded samples at resonance frequencies are shown in figure 6 . The response of the metallic part of the sensor includes branded gasoline is stronger and penetrates deeper with respect to unbranded gasoline case due to the lower real dielectric constant of branded gasoline sample. The surface current distributions for both samples are shown in figure 6 . It is observed that there are parallel and anti-parallel current distributions in the pattern that is responsible for the magnetic response. The two halves of rectangular metallic resonator behaves as two magnetic resonance circuits. The magnetic loop is complicated on the metallic parts and the space at the centre of the resonator. It is seen that the magnetic resonance of branded gasoline is also slightly higher than the unbranded one.

\section{Parametric study for MTM-based high efficiency portable sensor}

Furthermore, we have investigated the response of the proposed structure dimensions with parametric study. The resonator dimensions on the front side of MTM sensor ( $\mathrm{a} 3$ and a4 shown in figure 7) are changed for five different forms of structure. Reflection coefficient ( 222 in $\mathrm{dB}$ ) in numerical results are obtained for cases of (i) a $3=2.3 \mathrm{~mm}, \mathrm{a} 4=15 \mathrm{~mm}$, (ii) $\mathrm{a} 3=2.7 \mathrm{~mm}, \mathrm{a} 4=15.4 \mathrm{~mm}$, (iii) $\mathrm{a} 3=3.1 \mathrm{~mm}$, a4 $=15.8 \mathrm{~mm}$, (iv) a3 $=3.5 \mathrm{~mm}, \mathrm{a} 4=16.2 \mathrm{~mm}$ and (v) a3 $=4.3 \mathrm{~mm}, \mathrm{a} 4=17 \mathrm{~mm}$. As can be seen that both the long and short sides of rectangular patch are simultaneously increased. It is observed that the resonance frequency of reflection (S22 (dB)) shifts to lower frequencies with the increase of the front side resonator dimensions (a3 and a4 shown in figure 7) of the MTM-based sensor, since higher dimensions of resonator results in higher wavelength of EM wave to provide the resonance. It means lower frequency shift of the resonance depends on the resonator dimensions. These important results give chances to design a sensor to define the type of gasoline/diesel in desired frequency level. Also, it can be seen in the figure that reflection coefficients of numerical parametric study results (S22 in $\mathrm{dB})$ of MTM-based sensor structure are $-15.88,-17.78,-20.48,-24.87,-28.83 \mathrm{~dB}$, for five different forms of the structure. Hence, dimensions of (v) a $3=4.3 \mathrm{~mm}, \mathrm{a} 4=17 \mathrm{~mm}$ are used for the sensor application. The reduction of the reflection magnitude can be better impedance matching between free space and overall structure. It is well known that the reduction of reflection means better impedance matching between adjacent mediums. The 
proposed MTM-based sensor structure can be mechanically adopted for desired resonance frequencies.

\section{Conclusion}

In summary, a simple, cost effective and efficient MTMbased fuel-oil sensor for determining the difference between branded and unbranded gasoline and diesel samples is designed, fabricated and validated. Dielectric constant and loss tangent values of the branded and unbranded fuel-oil/diesel samples are measured by using a dielectric probe with a vector network analyzer for the MTM sensor structure designed in X-band. It is observed that there is $12 \mathrm{MHz}$ resonance shift for gasoline samples with a reflection constant of $6 \mathrm{~dB}$ and $72 \mathrm{MHz}$ resonance shift for diesel samples with a reflection constant of $4 \mathrm{~dB}$. It is concluded that the suggested sensor structure works effectively for distinguishing branded and unbranded fuel-oil/diesel samples and have mechanical tunability feature by tuning and/or by re-scaling their dimensions. Besides, the proposed sensor has a structure that can be fabricated and configurable easily for any desired frequency range allowing us to design sensors for other liquids having different EM behaviours in various microwave bands. Although the sensor was measured and its working mechanism was validated via a vector network analyzer, it can be used as a portable device by adding an RF source and a small size corresponding software. It is also planned by the authors to adjust the working frequency of the proposed design to work with cell phones, which has enough computing power and adequate features to operate this kind of sensor in portable sense.

\section{References}

[1] Sabah C 2012 Opt. Commun. 2854549
[2] Sabah C, Tastan H T, Dincer F, Delihacioglu K, Karaaslan M and Unal E 2013 Prog. Electromagn. Res. 138293

[3] Fang N, Lee H, Sun C and Zhang X 2005 Science 308534

[4] Sabah C 2010 Prog. Electromagn. Res. B 22341

[5] Sabah C 2010 Prog. Electromagn. Res. B 25225

[6] Dincer F, Karaaslan M, Unal E and Sabah C 2013 Prog. Electromagn. Res. 141219

[7] Dolling G, Wegener M, Soukoulis C M and Linden S 2007 Opt. Lett. 3253

[8] Tao H, Strikwerda A C, Liu M, Mondia J P, Ekmekci E, Fan K et al 2010 Appl. Phys. Lett. 97261909

[9] Xu X, Peng B, Li D, Zhang J, Wong L M, Zhang Q et al 2011 Nano Lett. 113232

[10] He X J, Qui L, Wang Y, Geng Z X, Wang J M and Gui T L 2011 Infrared J. Millim. Terahertz Waves 32902

[11] Chang Y T, Lai Y C, Li C T, Chen C K and Yen T J 2010 Opt. Express 189561

[12] Jakši'c Z, Vukovi'c S, Matovic J and Tanaskovi'c D 2011 Materials 41

[13] Lee H J, Lee J H and Jung H I 2011 Appl. Phys. Lett. 99163703

[14] Melik R, Unal E, Perkgoz N K, Puttlitz C and Demir H V 2009 Appl. Phys. Lett. 95011106

[15] Melik R, Unal E, Perkgoz N K, Puttlitz C and Demir H V 2009 Appl. Phys. Lett. 95181105

[16] Melik R, Unal E, Perkgoz N, Santoni B, Kamstock D, Puttlitz $\mathrm{C}$ et al 2010 IEEE J. Sel. Top. Quantum Electron. 16450

[17] Melik R, Unal E, Perkgoz N K, Puttlitz C and Demir H V 2010 Opt. Express 185000

[18] Arritt B, Adomanis B, Khraishi T and Smith D 2010 Appl. Phys. Lett. 97191907

[19] Pryce M, Aydin K, Kelaita Y A, Briggs R M and Atwater H A 2011 Philos. Trans. R. Soc. Lond. A 3693447

[20] Al-Hanib A I, Jansen C and Koch M 2008 Appl. Phys. Lett. 93 083507

[21] O'Hara F, Singh R, Brener I, Smirnova E, Han J, Taylor A J et al 2008 Opt. Express 161786

[22] He X J, Wang Y, Wang J M and Gui T L 2010 Microsyst. Technol. 161735

[23] Clemens M and Weiland T 2001 Prog. Electromagn. Res. 32 65 